\title{
Influence of feed additives of humic nature on the formation of nonspecific adaptive response of the rats' hemostasis under the influence of stressors
}

\section{Stepchenko L., Diachenko L.}

Dnipro state agrarian and economic university, Dnipro, Ukraine, stepchenko2@gmail.com, linadyach@ukr.net

doi: 10.36291/HIT.2019.stepchenko.098

The promising area for today is the searching for natural substances that can reduce the negative impact of hypoxia and oxidative stress on the body and thus can increase nonspecific adaptive resistance and affect the lability of the hemostasis system. It is known, that humic substances do not have a toxic effect that can increase the level of resistance of the animal body [1-3], but their effect on the system of hemostasis under the conditions of stress factors of different genesis has not been studied. Therefore, the aim of the study was to investigate the effect of feed additives of humic nature (FAHN) Humilid and Eco-Impulse Animal, which were obtained in different ways from the same peat on the state of the system of hemostasis of rats against the background of stress factors.

The studies were performed on white sexually mature young male rats weighing 180 $200 \mathrm{~g}$ based on vivarium. The animals were divided into 5 groups of 8 animals each. Group 1 (control) - intact animals. Rats 2, 3, 4 and 5 groups modeled combined stress. The model of water-immobilization stress in combination with elements of emotional stress (lengthening of daylight) was taken as a basis, due to which the effect of combined stress was achieved. The comparative analysis of the effect of the FAHN Humilid (acid-alkaline hydrolysis) and Eco-Impulse Animal (alkaline hydrolysis of peat using electric shock) on the overall parameters of the hemostasis system. Results were statistically analyzed using the onefactor dispersion analysis ANOVA. For all statistical calculations, the significance was considered as the value of $\mathrm{P}<0.05$. It has been established that due to waterimmobilization combined stress in rats, a significant prolongation of prothrombinase complex formation time, prothrombin activation time, and an increase in fibrinogen content compared to intact animals. In rats that received the FAHN Humilid after a stressful period, normalization of the formation time of the prothrombinase complex was observed, both externally and internally. In addition, in these rats, the time of prothrombin activation and the amount of fibrinogen did not differ from the control values. In turn, rats treated with EcoImpulse Animal FAHN showed stabilization of prothrombinase complex formation externally, whereas indicators characterizing the intrinsic pathway of prothrombinase formation, prothrombin activation time, and fibrinogen count did not differ from that of animals who did not receive any feed additives. Therefore, humic substances in the composition of feed additives Humidil can act as activators of faster formation of nonspecific adaptation of the hemostasis system to the action of stressors. The Eco-Impulse Animal, obtained by the specific method, exerts its influence only at the first stage of coagulation hemostasis. It is possible that upon receipt of this $\mathrm{FAHN}$, substances that have not been used in the processes of plasma factor synthesis or that have an inhibitory effect on the course of hemostasis reactions may have been formed.

References

1. Stepchenko L.M. Biologically active substances humic nature as a regulator of homeostasis of poultry. Collection of conference materials. 2011. 1:164-166.

2. Diachenko L.M., Stepchenko L.M. // Theor. Appl. Veter. Med. 2018. 6(3):34-38.

3. Paronik V.A. et al. // Animal Biology. 2015. 17(4):109-114. 